\title{
4. Clinical Evaluation of Arterial Administration of SMANCS in Oily Contrast Medium for Liver Cancer
}

\author{
Seiki Tashiro, MD and Hiroshi Maeda, Ph D, MD* \\ The First Department of Surgery and *Department of Microbiology, \\ Kumamoto University Medical School, Kumamoto
}

We have found that the lipid lymphographic agent, Lipiodol Ultra-Fluid (LPD, ethyl ester of the fatty acid of poppy seed oil; $38 \%$ in iodine content by weight, from Gurbert Laboratory, France), remained selectively in liver cancer when injected into the hepatic artery without ligation. Based on this finding, a new therapeutic approach to the treatment of liver cancer was attempted using a high-molecular-weight anticancer agent, SMANCS, dispersed and solubilized in LPD. SMANCS is a chemical conjugate of a synthetic copolymer of styrene-maleic acid and the proteinaceous antitumor agent neocarzinostatin linked by carbamide bond. SMANCS are soluble in some organic solvents such as pyridine and acetone, and also in LPD. The homogenous suspension of SMANCS in LPD, designated as SMANCS/LPD, was used for the present arterial administration.

One hundred and twenty-four patients with primary hepatoma and metastatic liver cancer, established either histologically or clinically, were subjected to the present study. Among these patients, 94 patients had primary hepatoma (69 patients had unresectable and 25 patients had resectable hepatoma), and 30 patients had metastatic liver cancer (25 patients had unresectable and 5 patients hasd resectable one). Usually, $1 \mathrm{mg}$ of SMANCS was dissolved in $1 \mathrm{ml}$ of LPD and the agent was injected at an average dose of $3.0-4.0 \mathrm{ml}$ via the celiac (62 times), the common hepatic (128 times), the proper hepatic (38 times) or the other peripheral arteries (34 times) by Seldinger's method. The total dose for each patient varied from $2 \mathrm{mg}$ to $25 \mathrm{mg}$. The optimum dose regimen was determined on the bases of staining in the CT scan and plain X-ray film findings after administration of the drug. Most patients received 2 to 4 arterial administrations given at intervals of 3 to 8 weeks.

A remarkable accumulation of LPD was clearly demonstrated in the tumors of all the patients with hepatoma and metastatic liver cancer, especially on X-ray CT scan. The amount of LPD retained in the tumor appeared to parallel with amount of neovascularity. The image of CTscan was characteristic to the types of liver cancer. LPD stayed densely in the entire tumor region in the primary hepatoma, whereas it stayed predominantly in the peripheral portion of the metastatic liver cancer. The latter type account about $70 \%$ of the metastatic liver cancer. Thus, the method was found useful for the diagnostic purpose.

The biological activity of SMANCS in the resected specimens of hepatoma and metastatic liver cancer persisted even after 2 months in the cancerous regions as well as the adjacent tissues where LPD was not recognized by softex. Both serum alpha-fetoprotein level and tumor size decreased in $91 \%$ and $90 \%$ of evaluable patients with hepatoma, respectively. Decreases of carcinoembryonic antigen level and tumor size occurred in $87 \%$ and $61 \%$ of evaluable patients with metastatic liver cancer after the treatment, respectively.

The histological examination of the resected specimens in 21 out of 25 patients with primary hepatoma and 4 out of 5 patients with metastatic hepatoma revealed that extensive necrosis of the tumor had occurred. This, however, did not accompany a drastic decrease in the tumor size.

While it is too early to evaluate longer term prognoses at this point, we have compared our present date with a comparison group of patients with advanced primary and metastatic 
liver cancer not treated with this protocol. The comparison group of 32 patients with unresectable primary hepatoma who were treated similarly to ligation of the hepatic artery and/or intraarterial continuous or single administration of mitomycin $\mathrm{C}$ and/or 5-fluorourail, had an average survival period of 2.3 months. Similarly, the comparison group of unresectable metastatic liver cancer exhibited 3.7 months. The average survival period of patients treated under the present protocol was more than 9.5 months (1 year survival: $49 \%$ ). This value is definitely longer than the comparison group. For the metastatic liver cancer, the result appears more promising than that of the primary hepatoma.
The major side effect observed after arterial infusion of SMANCS/LPD was fever of $38-39^{\circ} \mathrm{C}$ $(48 \%)$, although most patients became afebrile within a few days. About $24 \%$ of the patients experienced dull pain in the upper abdomen which lasted for about $15 \mathrm{~min}$ after the infusion. Mild and transitory elevations of serum GOT and GPT were seen in about $26 \%$. No hematosuppression was observed.

In conclusion, arterial administration therapy with an oily anticancer drug (SMANCS/LPD) offers several advantages in both diagnosis and treatment of liver cancer, particularly for its selective delivery of the drug to the target tumor. The side-effects were all transitory and mild, and no severe complications were experienced.

\title{
5. Preoperative Hyperthermo-chemo-radiotherapy for Esophageal Carcinoma
}

\author{
-Analysis of 20 Cases \\ Keizo Sugimachi, MD, FACS, Hidenobu KaI, MD \\ and Kiyoshi INOKuchi, MD, FACS \\ The Second Department of Surgery, Faculty of Medicine, \\ Kyushu University, Fukuoka, Japan
}

\section{INTRODUCTION}

Hyperthermia alone does have an anti-tumor effect and hyperthermia combined with chemotherapy and irradiation has proved to be even more effective. Clinical application of hyperthermia is, however, greatly hindered by the lack of an appropriate apparatus for selective application of heat to the malignant tissues in the digestive tract. We designed endotract antennae for Radio frequency (RF) and Microwave (MW), for clinical application in cases of carcinoma of the esophagus. After preliminary work and testing in laboratory animals ${ }^{1)}$ we have preoperatively applied this procedure for patients with esophageal carcinoma. Detailed histopathological examinations were done on the resected specimens. The effectiveness of hyperthermia combined with chemotherapy and irradiation was clearly evident.

\section{TECHNIQUE OF HYPERTHERMIA}

We designed antennae for $\mathrm{MW}(915 \mathrm{MHz})$ and RF (13.56 MHz), which can be introduced with an endoscope. As these antennae have a built-in thermometer, the exact temperature is easily monitored. When this antenna is inserted into the esophagus, then metastatic lymph nodes can be properly warmed.

As $\mathrm{RF}$ is more potent than MW, when the esophageal carcinoma was longer than $4 \mathrm{~cm}$, the antenna for RF was applied. Conversely, when the tumor is relatively small, the antenna for MW was applied.

In case of RF heating, a wide extracorporeal electrode measuring $30 \mathrm{~cm} \times 10 \mathrm{~cm}$ was applied 